\title{
Intensity modulated radiotherapy in carcinoma cervix with metastatic para-aortic nodes: an institutional experience from a Regional Cancer Centre of Eastern India
}

\author{
Misra Biplab, Maji Tapas, Lahiri Debarshi, Roy Sanjoy, Chaudhuri Prabir, Ray K. Dilip \\ Department of Radiotherapy, Chittaranjan National Cancer Institute, Kolkata, India
}

\begin{abstract}
Background: Cervical cancer is a major health problem, especially in developing countries like India. Extended field radiotherapy (EFRT) for cancer cervix treatment remains a challenging task for radiation oncologists. In the last decade studies have shown that EFRT using intensity modulated radiotherapy (IMRT) is feasible in treating gynaecological malignancies but there is a dearth of literature on this specific topic from this part of the world where patient profile differs greatly in several aspects from that of the western world. The aim of the study was evaluation of treatment response and toxicity profile in cases of carcinoma cervix with metastatic para-aortic nodes treated with intensity modulated radiotherapy technique.

Materials and methods: In this retrospective study the treatment records of 45 para-aortic node positive cervical cancer patients treated with EFRT (IMRT) and concurrent cisplatin were analysed for evaluation of loco-regional control and toxicities. Results: Forty-four patients received full course of treatment. Among those 44 patients, $93.2 \%$ achieved complete response. Overall, the treatment was tolerated well and toxicities were within acceptable limits. Acute grade 3-4 toxicities were observed mostly in the form of anaemia and leucopenia. Most common late toxicities were those of small and large intestine.

Conclusion: EFRT with concurrent chemotherapy was successfully delivered for para-aortic nodes positive cervical cancer patients in Indian scenario where under-nutrition, infection, anaemia and several other factors adversely influence treatment outcome. Pelvic and para-aortic control rates were satisfactory. The technique was associated with an acceptable acute and late toxicity profile.
\end{abstract}

Key words: cervical cancer; para-aortic lymph node; extended field radiotherapy; IMRT

Rep Pract Oncol Radiother 2021;26(3):400-407

\section{Introduction}

Cervical cancer is a major health problem among women worldwide with developing countries contributing $85 \%$ of all cases [1,2]. India also reflects a similar picture as cancer cervix is the second most common cancer in women in India with high prevalence in rural areas [3]. Due to the inadequacy of population-based screening programs, lesser ac- cess to proper healthcare facilities in rural areas, lack of health education and awareness, especially among the high-risk groups, $70-80 \%$ cases of cervical cancer present in advanced stages (stage III and IV) in India [4]. Along with the local extent of disease, nodal status also greatly influences the treatment strategy and outcome. Probability of lymph node involvement increases as the clinical stage advances. Nelson et al. showed that the incidence

Address for correspondence: Lahiri Debarshi, Specialist, Department of radiotherapy, Chittaranjan National Cancer Institute, 37 S P Mukherjee Road, Kolkata - 700026, India, tel: +91 9051513564; e-mail: debarshil@gmail.com 
of metastasis to para-aortic lymph nodes (PALN) in stage IIB cancer cervix was $16 \%$, whereas in stage IIIB it was as high as $46 \%$ [5]. Para-aortic lymph node metastasis itself is a poor prognostic factor for cervical cancer. Moreover, when these patients are treated with External Beam Radiotherapy (EBRT) up to the PALN level, the total irradiated volume and dose to the organs at risk increase significantly compared to pelvis only irradiation, resulting in added morbidity and making it a challenging task for the radiation oncologists.

In RTOG 79-20 trial (1995), it was shown that extended field radiotherapy (EFRT) improved overall survival (OS) in cervical cancer compared to standard pelvis only irradiation ( $55 \%$ vs. $44 \%$, $\mathrm{p}=.02$ ) but grade 4 and 5 toxicities were significantly higher in the EFRT arm as compared to the pelvis only arm ( $8 \%$ vs. $4 \%)$. Locoregional control rate was similar in both arms [6]. Many other studies also reported more or less similar results $[7,8]$ and, consequently, till date prophylactic para-aortic nodal irradiation (EFRT) is not a standard recommendation. On the other hand, few other studies showed that patients with documented spread to PALN can potentially become long-term survivors following locoregional radiotherapy [9, 10]. As a result, EFRT has become a standard of care for cervical cancer patients with documented PALN involvement, whenever feasible, after careful assessment of risk and benefit. Goodman et al. compiled survival statistics on cervical cancer patients with para-aortic lymph node metastasis and found an average 5-year survival rate of approximately $40 \%$ when treated with EFRT alone or EFRT plus chemotherapy [11].

In 1999, the National Cancer Institute (NCI) of the United States of America recommended cisplatin based chemotherapy concurrent with radiation, as the standard treatment protocol for cervical cancer patients, who require radiation. All of those trials [12-16], based on which the NCI recommendation was established, excluded patients with para-aortic lymph node metastasis. So, based on these trials, no logical conclusion could be drawn about the effectiveness of concurrent chemo-radiotherapy (CCRT) in improving the survival of patients with para-aortic node metastasis.

In the pre-Intensity Modulated Radiotherapy (IMRT) era, several large trials have been conducted to assess treatment response and toxicity in cervical cancer patients with metastatic para-aortic lymph node treated with EFRT, using four field box or opposed anterior-posterior beam and concurrent chemotherapy followed by brachytherapy. In those trials, the dose of external beam radiotherapy (EBRT) to PALN ranged from 45-50.4 Gy with subsequent boost (in some trials) to involved nodes up to 54-59 Gy. Although, the survival rates were about $30-40 \%$, the incidences of acute grade $3 / 4$ toxicities $(20-80 \%)$ and late severe toxicities (7-20\%) were quite high in these studies [17-21].

Since the first decade of the twenty-first century, the feasibility of IMRT is being explored in the treatment of cervical cancer with the hope of improved tumour control and lesser radiotherapy associated toxicity. In a survey in 2002, $15 \%$ of IMRT users reported treating a gynaecology patient with this technique [22]. In the follow-up survey in 2004, this percentage increased to $35 \%$ [23]. That data itself was a statement about the impact of IMRT in the treatment of gynecologic cancers.

The role of EF-IMRT for advanced cervical cancer was also being evaluated during that time with an aim to limit the treatment-related toxicities in various centres with promising results. In a dosimetric study of extended-field IMRT (EF-IMRT), Portelance et al. [24] showed better normal tissue sparing without changes in the target coverage. Gerszten et al. [25] conducted a study with 22 patients (2 having PALN metastasis) and concluded that EFRT using IMRT was quite feasible in treating gynecologic malignancies as did Salama et al. [26] with their experience of treating 13 such cases.

Beriwal et al. [27] conducted a similar study to assess early clinical outcomes with concurrent cisplatin and EF-IMRT for carcinoma of the cervix with 36 patients including 10 having positive PALN. The treatment was tolerated well with a good loco-regional control rate (34 patients achieved complete response), with distant metastases being the predominant mode of failure (2 infield recurrence and 9 distant metastases).

An electronic search of the PubMed database was performed to obtain key literature on this topic but only a few were found from this part of the world [28]. Like most other developing countries, India also has relatively few public cancer hospitals with IMRT facilities at present, with our centre being one of them, and which have used IMRT in this setting. Hence, the assessment of feasibility and 
efficacy of IMRT in the treatment of PALN positive cervical cancer in the Indian scenario was taken up for investigation in this retrospective study with the primary objective of evaluating locoregional control and toxicity (acute and late).

\section{Materials and methods}

This was a retrospective observational study conducted at a tertiary cancer hospital in India. The treatment records of 45 para-aortic node positive, histopathologically proven cervical cancer patients aged between 20-65 years with Karnofsky Performance Score $\geq 60$, normal hemogram and biochemical profiles at baseline and treated with EFRT plus weekly concurrent cisplatin during the period of June 2012 to June 2015, were analysed from the hospital database. Nodal status was assessed by CT-Scan only (as the institute lacked a PET-CT Scan facility). No surgical assessment of nodes was done and patients having nodes larger than $3 \mathrm{~cm}$ were excluded from the study. Due approval of the institutional ethics committee was taken before analysing the data.

The prescribed dose of external beam radiotherapy (EBRT) was 50.4 Gy in 28 fractions to the Planning Target Volume (PTV) along with weekly cisplatin $\left(40 \mathrm{mg} / \mathrm{m}^{2}\right)$. No nodal boost was given as the optimal dose for acceptable toxicities is not well established in the Indian patient population. Dose constraints to organs at risk were prescribed as follows: kidney - mean dose < 15-18 Gy, spinal cord $-\mathrm{D}_{\max }<50 \mathrm{~Gy}$, bowel bag - V $45<195 \mathrm{cc}$, liver mean dose $<30$ Gy, femoral head $-\mathrm{V} 50<5 \%$. All patients were planned to receive 4 fractions of weekly intracavitary High Dose Rate (HDR) $\operatorname{Ir}^{192}$ Brachytherapy starting from the $4^{\text {th }}$ week of EBRT. Dose per fraction of HDR intracavitary brachytherapy was 6 Gy calculated at the conventional point A as per our institutional practice. All patients were reviewed meticulously for assessment of any radiation reaction or chemotherapy related toxicity during the whole course of treatment.

CT-simulation for all patients was done with a full bladder protocol and in a supine position.

Nodal contouring: Clinical target volume for lymph nodes (CTV-N) included para-aortic, common iliac, external iliac, internal iliac, obturator and presacral lymph nodes. Para-aortic nodal contouring was started at the level of celiac trunk.
Involved nodes were contoured as nodal gross tumour volume (GTV-N) and $1 \mathrm{~cm}$ margin was given around GTV-N to draw CTV-N. Uniformly, blood vessels were given a margin of $7 \mathrm{~mm}$ to draw the nodal CTV for pelvic nodes. The contour was extended around common iliac vessels posteriorly and laterally so as to include connective tissue between iliopsoas muscles and lateral surface of the vertebral body.

Contouring of different volumes for the primary tumour: CTV Primary (CTV-P) included GTV Primary, uterine cervix, uterine corpus, parametrium, vagina and ovaries. For the vagina, paravaginal tissue was included along with the vaginal wall. The lower extent of disease was marked with a fiducial marker during CT-simulation and CTV-P was contoured up to $2 \mathrm{~cm}$ below this marker. For parametrium, the cranial border was defined at the level where the true pelvis began. Superiorly, contouring was stopped once loops of bowel were seen next to the uterus. Anteriorly, delineation was done up to the posterior border of the bladder in the central region, while, in the periphery, it extended up to the anterior end of the lateral pelvic bony wall. Posteriorly, the parametrium was contoured only up to the anterior part of the mesorectal fascia. In the case of significant parametrial invasion (stage IIIB) or uterosacral ligament involvement, the entire mesorectum was included in CTV-P. Laterally, the parametrium was contoured up to the medial edge of internal obturator muscle. Caudal border of the parametrium was taken at the medial border of the levatorani or at the pelvic floor.

Planning target volume (PTV) margin: the uterine motion was accounted for by giving an asymmetric PTV margin over CTV-P, with $15 \mathrm{~mm}$ antero-posteriorly, $15 \mathrm{~mm}$ supero-inferiorly and 10 $\mathrm{mm}$ laterally. All plans were done by using 7 to 9 coplanar beams (6 MV photons). Collimator angle of 45 degrees was given for better coverage (Fig. 1).

Patient's position was verified with cone-beam computed tomography (CBCT) during the course of treatment to check the reproducibility of patient set-up.

\section{Statistical analysis}

Descriptive statistical analyses were performed to calculate the means with corresponding standard deviations (SD). Test of proportion was used to find the standard normal deviate $(\mathrm{Z})$ and 


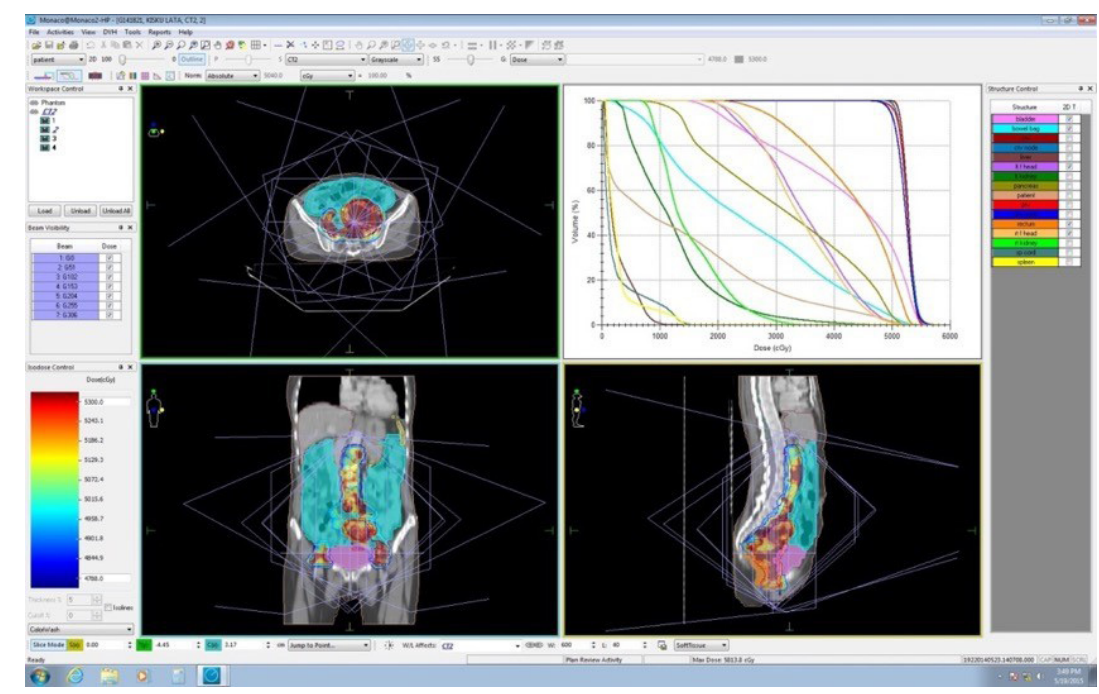

Figure 1. Beam arrangement and dose painting (from treatment planning system)

Chi-square $\chi^{2}$ test was performed to find the associations. $\mathrm{P} \leq 0.05$ was taken to be statistically significant. Kaplan-Meier survival analysis and Log Rank test were performed to assess the overall survival/disease-free survival and to compare the same between two independent groups, respectively.

Follow up: At 1 month after completion of the full course of treatment, all the patients had their first evaluation to assess the treatment response clinically and by CT-Scan of the abdomen and pelvis. The response was assessed using response evaluation criteria in solid tumours (RECIST version 1.1) [29]. Thereafter, the clinical follow up continued every 3 months for the first year and then at 6-month intervals. Appropriate investigations, including imaging (CT-Scan/MRI/ PET-CT Scan) and definitive histopathological assessment, were done in suspected cases of recurrent tumour as per tumour board decision during the course of follow-up.

\section{Results}

\section{Patient characteristics}

The mean age (mean $\pm \mathrm{SD}$ ) of the patients was $52.93 \pm 5.80$ years with the range of 38-64 years. Baseline patients' characteristics are given below in Table 1.

\section{Treatment response}

Forty-four (44) patients received the full course of radiotherapy except for one patient who did not receive the last fraction of intracavitary brachytherapy as she had persistent central disease with pelvic nodes during that time and developed a systemic infection and renal failure subsequently. Thirty-five

Table 1. Baseline patients' characteristic

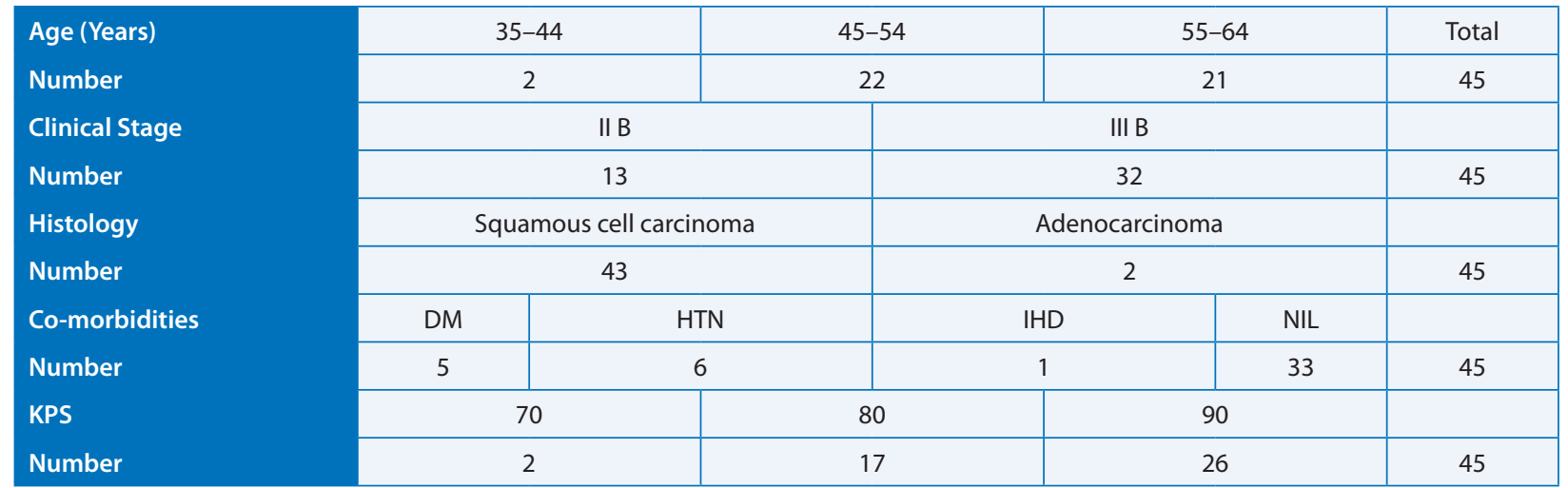

DM - diabetes mellitus; HTN — hypertension; IHD - ischemic heart disease 


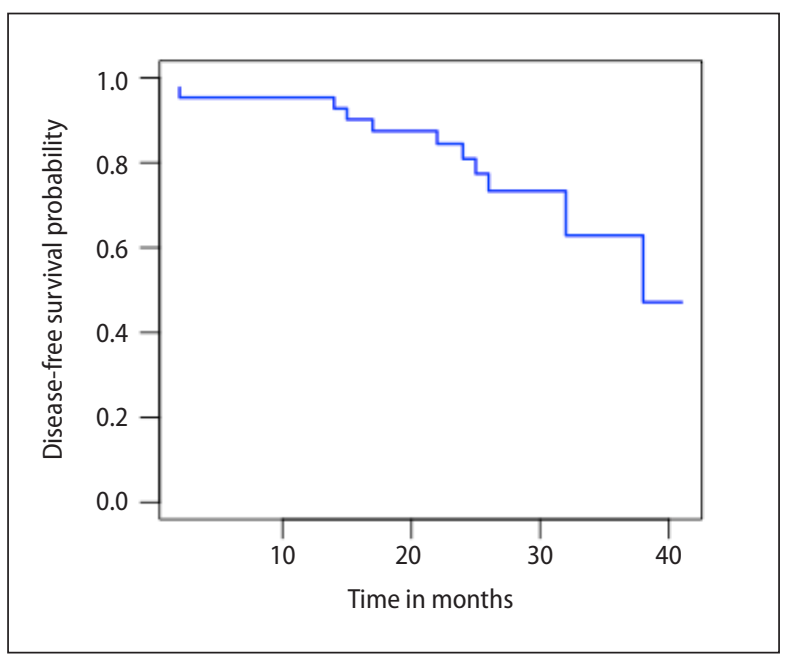

Figure 2. Disease free survival curve

patients (77.8\%) received all 6 cycles of chemotherapy (weekly cisplatin $40 \mathrm{mg} / \mathrm{m}^{2}$ ), whereas 5 cycles and 4 cycles of chemotherapy were received by 4 and 6 patients, respectively. Leucopenia, anaemia, nausea and vomiting were the main reasons for discontinuation of chemotherapy.

Among those 44 patients who completed radiotherapy, 41 patients (93.2\%) achieved complete response. Two among the remaining three patients who showed partial response had persistent para-aortic node (one of them with pulmonary metastases) and the other one had both pelvic and para-aortic nodes. So, para-aortic nodal control was $93.2 \%$, which was a satisfactory outcome.

The duration of median follow up was 26 months (range 2-41 months). Mean and 2-year disease free survival (DFS) were 21.19 \pm 9.91 months and $51.16 \%$, respectively (Fig. 2). The log-rank test showed that there was no significant difference in the disease-free survival pattern of clinical stage IIB and stage IIIB patients ( $p>0.05)$. Twelve (12) patients (29.27\%) presented with recurrent disease within the irradiated field or distant metastases during the subsequent course of follow-up. Among those 12 patients, 3 patients $(7.32 \%)$ had central disease recurrence ( 2 in the cervix, 1 in the anterior wall of the rectum); 2 patients $(4.87 \%)$ had recurrent para-aortic nodes; 3 had a recurrence in the pelvic nodes (among those one had vertebral metastases) and 4 patients (9.76\%) developed isolated distant metastases outside the irradiated field.

Mean and 2-year overall survival (OS) were $24.44 \pm 9.21$ months and $67.44 \%$, respectively, and

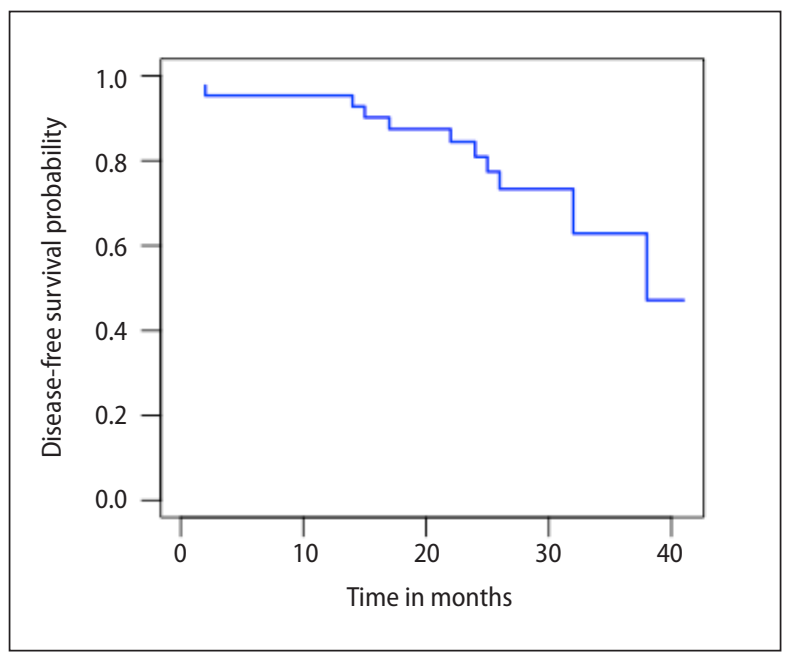

Figure 3. Overall survival curve

there was no significant difference in the overall survival pattern of clinical stage IIB and stage IIIB patients $(\mathrm{p}>0.05)$ (Fig. 3).

\section{Toxicity profile}

Overall, the whole course of treatment was tolerated well by most of the patients. Toxicities observed during treatment and within the first 3 months of completion of treatment were considered as an acute toxicity and beyond that as late toxicity.

Acute grade 3 or grade 4 toxicities were observed mostly in the form of anaemia and leucopenia. Both grade 3 anaemia and leucopenia were seen in $10 \mathrm{pa}-$ tients $(22.2 \%)$ and only one patient suffered from grade 4 leucopenia. Two (2) patients (4.4\%) had incidence of acute grade 3 skin reaction, whereas the incidence of acute grade 3 toxicity for upper GI, lower GI and pelvis, and the genitourinary system was minimal (2.2\%) (Tab. 2).

Thirty-two (32) patients (72.73\%) completed the overall treatment within 8 weeks duration. Twenty (20) patients (44.4\%) needed treatment break, but among those only 3 patients (15\%) suffered treatment interruption of more than 7 days. Haematological toxicities followed by nausea and vomiting were the most common causes of treatment interruption.

Till the date of the last follow-up, most common late toxicities were those of the small and large intestine followed by the urinary bladder. Incidences of grade 3 or grade 4 late toxicities were observed in 4 patients (9.1\%). Two (2) patients suffered grade 4 
Table 2. Acute toxicity profile

\begin{tabular}{|l|c|c|c|c|c|c|}
\hline & Grade 0 & Grade 1 & Grade 2 & Grade 3 & Grade 4 & Total no. of patient \\
\hline Acute upper Gl toxicity & 15 & 19 & 10 & 1 & 0 & 45 \\
\hline Acute lower Gl and pelvic toxicity & 5 & 16 & 23 & 1 & 0 & 45 \\
\hline Anaemia (Hb \%) & 5 & 11 & 19 & 10 & 0 & 45 \\
\hline Leucopenia & 8 & 12 & 14 & 10 & 1 & 45 \\
\hline Acute toxicity of skin & 5 & 18 & 20 & 2 & 0 & 45 \\
\hline Acute genitourinary toxicity & 15 & 19 & 10 & 1 & 0 & 45 \\
\hline
\end{tabular}

Table 3. Late toxicity profile

\begin{tabular}{|c|c|c|c|c|c|c|}
\hline & Grade 0 & Grade 1 & Grade 2 & Grade 3 & Grade 4 & Total No. of Patient \\
\hline Bladder & 34 & 7 & 2 & 1 & 0 & 44 \\
\hline Small/Large intestine & 28 & 8 & 5 & 1 & 2 & 44 \\
\hline Kidney & 41 & 2 & 1 & 0 & 0 & 44 \\
\hline Skin & 36 & 8 & 0 & 0 & 0 & 44 \\
\hline
\end{tabular}

bowel toxicity (1 fistula and 1 sub-acute intestinal obstruction), one (1) patient showed the features of grade 3 bowel toxicity and one had grade 3 bladder toxicity (Tab. 3).

\section{Discussion}

While the potential for normal-tissue sparing is the main motivation behind the implementation of IMRT for cervical cancer, achieving good target coverage remains the primary objective. Consistent and accurate target volume definition as per available guidelines was essential as the salvage treatment for relapsed disease due to a geographic miss is rarely successful [30]. The target volumes contoured in the pelvis were generous, accounting for the inability to clearly delineate the areas at risk by CT criteria. The volumes closely approximated the large pelvic fields traditionally used to treat cervical cancer but the extended field portion, covering the para-aortic nodes, was less generous. As the PTV margins were relatively tight, the help of image guidance during the delivery of radiotherapy was taken.

We prescribed 50.4 Gy to PTV in 28 fractions with no simultaneous or subsequent boost to involved nodes. In the pre IMRT era, several authors treated PALN in cervical cancer patients by conventional EFRT with concurrent chemotherapy. Among those studies, Grisby et al. [17] (RTOG 92-01) and Small W Jr et al. [18] (Arm 1 of RTOG 0116) treat- ed nodal PTV with 48 Gy and 45 Gy, respectively, followed by a boost to the involved nodes up to 54-59.4 Gy. They reported very high rates of RTOG G3/G4 toxicities. Varia et al. [19] treated PALN with $45 \mathrm{~Gy}$ and no subsequent boost to involved nodes and managed to achieve similar treatment outcome with a less severe toxicity profile.

In the IMRT era, Gerszten et al [25]. and Beriwal et al. [27] used the simultaneous integrated boost to treat the involved nodes with dose up to 55-60 Gy while prescription for whole nodal PTV was $45 \mathrm{~Gy}$ in 25 fractions. Both authors reported good disease control with an acceptable toxicity profile.

A large proportion of our patients (44.4\%) had suffered acute RTOG grade 2 skin toxicity which was a bit higher than those reported in the previously mentioned trials. The difference was most likely due to poor maintenance of hygiene by our patients and the hot and humid weather in this part of the world. Other acute and late toxicities were comparable to those reported in the western population.

We implemented HDR intracavitary brachytherapy, using 2-dimentional fluoroscopic image guidance, from the fourth week of EBRT to cut short the overall treatment time. Most of our patients (55.56\%) needed no treatment interruption and 32 patients $(72.73 \%)$ completed the overall treatment within 8 weeks.

Although haematological toxicities were the most common cause of treatment interruption, the 
overall results were quite satisfactory despite the relatively compromised nutritional and immune status of our patients. The pelvis and spine contain a significant volume of bone marrow tissue and irradiation of these regions has a known impact on peripheral blood counts. IMRT may also allow significant sparing of bone marrow.

We could not use PET-CT scan for diagnosis, treatment planning or response assessment as our institution did not have PET-CT scan facility at that time and most of our patients could not afford a PET-CT Scan in a private hospital. This is a limitation of this study but CT based assessment still is the major option in resource constrained settings.

We may consider intensifying this so far well tolerated treatment regimen by adding simultaneous/sequential boost dose to involved nodes, preferably by PET-CT based planning, for any further improvement in loco-regional control in the future.

\section{Conclusion}

In this retrospective observational study, an intensity-modulated radiotherapy technique was used to deliver extended field radiotherapy with concurrent chemo-sensitization for para-aortic nodes-positive cervical cancer patients in the Indian scenario, where undernutrition, infection, anaemia, and several other socio-economic factors adversely influence successful treatment delivery and treatment outcome. Pelvic and para-aortic nodal control rates were comparable to those in the literature. The treatment was associated with an acceptable acute and late toxicity profile without significant treatment prolongation. The impact of simultaneous nodal boost (using PET-CT based planning) may be assessed to determine its role in further improving disease outcome among the Indian patient population. This potential role for IMRT merits further evaluation with larger patient numbers and longer follow-up, preferably with a prospective randomized control study.

\section{Conflict of interest}

None declared.

\section{Funding}

This study has not been funded by any agency and there is no financial conflict of interest.

\section{Ethics approval and consent to participate}

Due ethical approval and written informed consent from all the participants were taken for the study. Institutional Ethics Committee approval reference number- CNCI/IEC/A-4.311/2012/2(C).

\section{Acknowledgement}

The authors sincerely thank the patients and all the staffs of the Department of Radiotherapy, Chittaranjan National Cancer Institute, Kolkata.

\section{References}

1. International Agency for research on cancer. Cervical cancer estimated incidence, mortality and prevalence worldwide in 2012. World Health Organisation; 2012. http://globocon.iarc.fr/pages/factsheetcancer. aspx?Cancer=cervix (12th May 2012).

2. Jemal A, Bray F, Center MM, et al. Global cancer statistics. CA Cancer J Clin. 2011; 61(2): 69-90, doi: 10.3322/ caac.20107, indexed in Pubmed: 21296855.

3. Park K. Epidemiology of chronic non-communicable diseases and conditions. Park's Textbook of preventive and social medicine. 23rd Ed. Jabalpur, M/s Banarsidas Bhanot 2015: 388-389.

4. Basu P, Biswas J, Mandal R, et al. Is interferon-alpha and retinoic acid combination along with radiation superior to chemo-radiation in the treatment of advanced carcinoma of cervix? Indian J Cancer. 2006; 43(2): 54-59, doi: 10.4103/0019-509x.25885, indexed in Pubmed: 16790941.

5. Nelson JH, Boyce J, Macasaet M, et al. Incidence, significance, and follow-up of para-aortic lymph node metastases in late invasive carcinoma of the cervix. Am J Obstet Gynecol. 1977; 128(3): 336-340, doi: 10.1016/00029378(77)90633-0, indexed in Pubmed: 860741.

6. Rotman M, Pajak TF, Choi K, et al. Prophylactic extendedfield irradiation of para-aortic lymph nodes in stages IIB and bulky IB and IIA cervical carcinomas. Ten-year treatment results of RTOG 79-20. JAMA. 1995; 274(5): 387-393, indexed in Pubmed: 7616634.

7. Haie C, Pejovic MH, Gerbaulet A, et al. Is prophylactic paraaortic irradiation worthwhile in the treatment of advanced cervical carcinoma? Results of a controlled clinical trial of the EORTC radiotherapy group. Radiother Oncol. 1988; 11(2): 101-112, doi: 10.1016/0167-8140(88)90245-9, indexed in Pubmed: 3281186.

8. Chatani M, Matayoshi Y, Masaki N, et al. Prophylactic irradiation of para-aortic lymph nodes in carcinoma of the uterine cervix. A prospective randomized study. Strahlenther Onkol. 1995; 171(11): 655-660, indexed in Pubmed: 7502230.

9. Lepanto P, Littman P, Mikuta J, et al.Treatment of para-aortic nodes in carcinoma of the cervix. Cancer. 1975;35(6): 15101513, doi: 10.1002/1097-0142(197506)35:6<1510::aidcncr2820350605>3.0.co;2-u, indexed in Pubmed: 807311.

10. Nori D, Valentine E, Hilaris BS. The role of paraaortic node irradiation in the treatment of cancer of the cervix. Int J Radiat Oncol Biol Phys. 1985; 11(8): 1469- 
1473, doi: 10.1016/0360-3016(85)90334-7, indexed in Pubmed: 4019270.

11. Goodman HM, Niloff JM, Nelson JR. et al. Cervical malignancies. In: Knapp RC, Berkowitz RS. ed. Gynecologic oncology. Macmillan, New York 1986: 225-273.

12. Keys HM, Bundy BN, Stehman FB, et al. Cisplatin, radiation, and adjuvant hysterectomy compared with radiation and adjuvant hysterectomy for bulky stage IB cervical carcinoma. N Engl J Med. 1999; 340(15): 1154-1161, doi: 10.1056/NEJM199904153401503, indexed in Pubmed: 10202166.

13. Peters WA, Liu PY, Barrett RJ, et al. Concurrent chemotherapy and pelvic radiation therapy compared with pelvic radiation therapy alone as adjuvant therapy after radical surgery in high-risk early-stage cancer of the cervix. J Clin Oncol. 2000; 18(8): 1606-1613, doi: 10.1200/ JCO.2000.18.8.1606, indexed in Pubmed: 10764420.

14. Whitney CW, Sause W, Bundy BN, et al. Randomized comparison of fluorouracil plus cisplatin versus hydroxyurea as an adjunct to radiation therapy in stage IIB-IVA carcinoma of the cervix with negative para-aortic lymph nodes: a Gynecologic Oncology Group and Southwest Oncology Group study. J Clin Oncol. 1999; 17(5): 1339-1348, doi: 10.1200/JCO.1999.17.5.1339, indexed in Pubmed: 10334517.

15. Rose PG, Bundy BN, Watkins EB, et al. Concurrent cisplatin-based radiotherapy and chemotherapy for locally advanced cervical cancer. N Engl J Med. 1999; 340(15): 1144-1153, doi: 10.1056/NEJM199904153401502, indexed in Pubmed: 10202165.

16. Eifel PJ, Winter K, Morris M, et al. Pelvic irradiation with concurrent chemotherapy versus pelvic and para-aortic irradiation for high-risk cervical cancer: an update of radiation therapy oncology group trial (RTOG) 90-01. J Clin Oncol. 2004; 22(5): 872-880, doi: 10.1200/JCO.2004.07.197, indexed in Pubmed: 14990643.

17. Grigsby PW, Heydon K, Mutch DG, et al. Long-term followup of RTOG 92-10: Cervical cancer with positive paraaortic lymph nodes. Int J Radiat Oncol Biol Phys. 2000; 51(4): 982-987, doi: 10.1016/s0360-3016(01)01723-0, indexed in Pubmed: 11704321.

18. Small W, Winter K, Levenback C, et al. Extended field iadiation and intacavitay bachytheapy combined with cisplatin chemotheapy fo cevical cance with positive paa-aotic o high common iliac lymph nodes: Result of Am 1 of RTOG 0116. Int J Radiation Oncology Biol Phys. 2007; 68(4): 1081-1087, doi: 10.1016/j.jijrobp.2007.01.026, indexed in Pubmed: 17398031.

19. Varia MA, Bundy BN, Deppe G, et al. Cervical carcinoma metastatic to para-aortic nodes: extended field radiation therapy with concomitant 5-fluorouracil and cisplatin chemotherapy: a Gynecologic Oncology Group study. Int J Radiat Oncol Biol Phys. 1998; 42(5): 1015-1023, doi: 10.1016/s0360-3016(98)00267-3, indexed in Pubmed: 9869224

20. Hacker NF, Wain GV, Nicklin JL. Resection of bulky positive lymph nodes in patients with cervical carcinoma. Int J Gynecol Cancer. 1995; 5(4): 250-256, doi: 10.1046/j.15251438.1995.05040250.x, indexed in Pubmed: 11578485.

21. Chou HH, Wang CC, Lai CH, et al. Isolated paraaortic lymph node recurrence after definitive irradiation for cervical carcinoma. Int J Radiat Oncol Biol Phys. 2001; 51(2): 442-448, doi: 10.1016/s0360-3016(01)01628-5, indexed in Pubmed: 11567819.

22. Mell LK, Roeske JC, Mundt AJ. A survey of intensitymodulated radiation therapy use in the United States. Cancer. 2003; 98(1): 204-211, doi: 10.1002/cncr.11489, indexed in Pubmed: 12833473.

23. Mell LK, Mehrotra AK, Mundt AJ. Intensity-modulated radiation therapy use in the U.S., 2004. Cancer. 2005; 104(6): 1296-1303, doi: 10.1002/cncr.21284, indexed in Pubmed: 16078260.

24. Portelance L, Chao KS, Grigsby PW, et al. Intensity-modulated radiation therapy (IMRT) reduces small bowel, rectum, and bladder doses in patients with cervical cancer receiving pelvic and para-aortic irradiation. Int J Radiat Oncol Biol Phys. 2001; 51(1): 261-266, doi: 10.1016/s03603016(01)01664-9, indexed in Pubmed: 11516876.

25. Gerszten K, Colonello K, Heron DE, et al. Feasibility of concurrent cisplatin and extended field radiation therapy (EFRT) using intensity-modulated radiotherapy (IMRT) for carcinoma of the cervix. Gynecol Oncol. 2006; 102(2): 182-188, doi: 10.1016/j.ygyno.2005.12.044, indexed in Pubmed: 16516281.

26. Salama JK, Mundt AJ, Roeske J, et al. Preliminary outcome and toxicity report of extended-field, intensity-modulated radiation therapy for gynecologic malignancies. Int J Radiat Oncol Biol Phys. 2006; 65(4): 1170-1176, doi: 10.1016/j. ijrobp.2006.02.041, indexed in Pubmed: 16730136.

27. Beriwal S, Gan GN, Heron DE, et al. Early clinical outcome with concurrent chemotherapy and extended-field, intensity-modulated radiotherapy for cervical cancer. Int J Radiat Oncol Biol Phys. 2007; 68(1): 166-171, doi: 10.1016/j. ijrobp.2006.12.023, indexed in Pubmed: 17321070.

28. Gupta M, Chopra S, Kunder S, et al. Early toxicity and treatment outcomes of extended field-intensity modulated radiotherapy for cervical cancer patients with para-aortic nodal metastasis. Ecancermedicalscience. 2019; 13: 957, doi: 10.3332/ecancer.2019.957, indexed in Pubmed: 31645885.

29. Ctep.cancer.gov.2020. https://ctep.cancer.gov/protocolDevelopment/docs/recist_guideline.pdf.

30. Hong JH, Tsai CS, Lai CH, et al. Recurrent squamous cell carcinoma of cervix after definitive radiotherapy. Int J Radiat Oncol Biol Phys. 2004; 60(1): 249-257, doi: 10.1016/j. ijrobp.2004.02.044, indexed in Pubmed: 15337563. 